\title{
Nanosized superparamagnetic precipitates in cobalt-doped $\mathrm{ZnO}$
}

\author{
Matthias Opel * Karl-Wilhelm Nielsen, Sebastian Bauer, \\ Sebastian T. B. Goennenwein, and Rudolf Gross \\ Walther-Meißner-Institut, Bayerische Akademie \\ der Wissenschaften, 85748 Garching, Germany \\ Júlio C. Cezar \\ European Synchrotron Radiation Facility, 38043 Grenoble Cedex 9, France \\ Dieter Schmeisser \\ Angewandte Physik II, Brandenburgische Technische \\ Universität Cottbus, 03046 Cottbus, Germany \\ Jürgen Simon and Werner Mader \\ Institut für Anorganische Chemie, Rheinische \\ Friedrich-Wilhelms-Universität Bonn, 53117 Bonn, Germany
}

(Dated: October 24, 2018) 


\begin{abstract}
The existence of semiconductors exhibiting long-range ferromagnetic ordering at room temperature still is controversial. One particularly important issue is the presence of secondary magnetic phases such as clusters, segregations, etc... These are often tedious to detect, leading to contradictory interpretations. We show that in our cobalt doped $\mathrm{ZnO}$ films grown homoepitaxially on single crystalline $\mathrm{ZnO}$ substrates the magnetism unambiguously stems from metallic cobalt nano-inclusions. The magnetic behavior was investigated by SQUID magnetometry, x-ray magnetic circular dichroism, and AC susceptibility measurements. The results were correlated to a detailed microstructural analysis based on high resolution x-ray diffraction, transmission electron microscopy, and electron-spectroscopic imaging. No evidence for carrier mediated ferromagnetic exchange between diluted cobalt moments was found. In contrast, the combined data provide clear evidence that the observed room temperature ferromagnetic-like behavior originates from nanometer sized superparamagnetic metallic cobalt precipitates.
\end{abstract}

PACS numbers: 75.25.+z 75.30.-m 75.50.Pp 78.70.Dm 


\section{INTRODUCTION}

Materials combining different functionalities have attracted huge interest due to the ever growing demands in applications. Regarding spintronics 122 , semiconductors that are ferromagnetic above room temperature (RT) are in the focus of current research, as they combine ferromagnetic exchange with versatile electronic properties. They certainly would pave the way to novel devices with new functionalities. Dilute magnetic semiconductors (DMS) in which itinerant charge carriers mediate a ferromagnetic coupling between the moments of diluted transition metal (TM) ions, have been considered as promising materials ${ }^{3}$. While ferromagnetism has first been observed in Mn-doped InAs $s^{4}$ and $\mathrm{GaAs}^{5}$ the Curie temperatures $T_{\mathrm{C}}$ in those materials are still well below RT. In contrast, RT ferromagnetism has been predicted for wide bandgap TM-doped semiconductors such as ZnO:TM or GaN:TM³ . Although these materials have been studied extensively during the last years their magnetic properties are still under debate. For $\mathrm{ZnO}$ :TM, hole-mediated ferromagnetism was originally suggested ${ }^{3}$ whereas in the majority of experimental studies RT ferromagnetism has been found in $n$-type material $1,7,8,9,10,11,12, \underline{13}$. In turn, recent theoretical models explain ferromagnetic coupling in terms of bound magnetic polarons $\frac{14,15}{4}$ or a ligand-to-metal charge transfer $\underline{16}, 17$. While a large number of experimental studies seem to provide evidence for carrier-mediated RT ferromagnetism in ZnO:TM, also the absence of any ferromagnetic coupling has been reported $18,19,20,21,22$, thereby further fueling the controversy about the occurrence and nature of ferromagnetism in ZnO:TM. Furthermore, calculations show a magnetic moment at oxygen rich surfaces in e.g. $\mathrm{ZrO}_{2}$ or $\mathrm{Al}_{2} \mathrm{O}_{3}{ }^{23}$ which even calls into question the necessity of TM-doping for ferromagnetism. Finally, unexpected magnetic coupling has also been found in nominally undoped oxides like $\mathrm{HfO}_{2}$ or $\mathrm{TiO}_{2} \underline{24}, 25$.

Another important issue concerning DMS is the formation of ferromagnetic clusters. While they have long been regarded as detrimental for spintronic applications it has recently been shown that they may be utilized to control high temperature ferromagnetism in semiconductors and to tailor spintronic functionalities 26 . TM-doped $\mathrm{ZnO}$ is known to form

nanosized (inter)metallic inclusions $27,28,29,30$ which may be responsible for the observed RT magnetic response. The same is true for other TM-doped semiconductors like Mn-doped $\mathrm{Ge}^{31,32}$. Therefore, an unambiguous clarification of the origin of magnetism requires a systematic combined magnetic and microstructural analysis. We have performed such a study 
on cobalt-doped ZnO. For magnetic characterization, we used SQUID magnetometry as well as x-ray magnetic circular dichroism (XMCD) and AC susceptibility measurements. The advantage of XMCD is the independent and element-specific determination of spin and orbital magnetic moments on an atomic level. In contrast to previous studies, we performed XMCD in both the total electron and fluorescence yield modes allowing to distinguish between surface and bulk magnetic properties. Our data provide clear evidence that the observed room temperature magnetism is not related to a bulk homogeneous DMS, but can rather be explained by the presence of superparamagnetic metallic cobalt precipitates. This is further confirmed by careful x-ray diffraction and high-resolution transmission electron microscopy, making the nanosized cobalt inclusions visible.

\section{SAMPLE FABRICATION}

The epitaxial $\mathrm{Zn}_{0.95} \mathrm{Co}_{0.05} \mathrm{O}$ thin films studied here were grown by pulsed laser deposition from a stoichiometric polycrystalline target using a KrF excimer laser (248 nm) at a repetition rate of $2 \mathrm{~Hz}$ with an energy density at the target of $2 \mathrm{~J} / \mathrm{cm}^{2}$. The thin films were deposited on single crystalline, $c$-axis oriented $\mathrm{ZnO}(0001)$ substrates at temperatures $T_{\mathrm{G}}$ between $300^{\circ} \mathrm{C}$ and $600^{\circ} \mathrm{C}$ in pure argon atmosphere at a pressure of $4 \times 10^{-3}$ mbar. During deposition, the film growth was monitored by in-situ RHEED ${ }^{33}$. The structural analysis of the samples was performed in a Bruker-AXS four circle diffractometer (D8 Discover) using $\mathrm{Cu} \mathrm{K} \mathrm{K}_{\alpha 1}$ x-ray radiation. High-resolution x-ray diffraction $(\omega-2 \theta)$ scans in out-of-plane direction and reciprocal space mappings of the (1011) reflection reveal an excellent crystalline quality of the films. The $c$-axis lattice parameters were found to range between 5.22 and $5.32 \AA$. The mosaic spread indicated by the full width at half maximum of the rocking curves of the (0002) reflection was as low as $0.02^{\circ}$. More details are given elsewhere ${ }^{11}$. As the samples were grown in the absence of oxygen they are highly conductive. Their room temperature resistivity is in the order of $1 \Omega \mathrm{cm}$. Although similar data have been found for all films grown at different temperatures, in the following we will focus on $\mathrm{Zn}_{0.95} \mathrm{Co}_{0.05} \mathrm{O}$ thin films grown at $T_{\mathrm{G}}=400^{\circ} \mathrm{C}$ and $500^{\circ} \mathrm{C}$ with a thickness of $350 \mathrm{~nm}$, for clarity. 


\section{MAGNETIC CHARACTERIZATION}

Magnetization and AC susceptibility were measured in a Quantum Design superconducting quantum interference device (SQUID) magnetometer (MPMS XL-7) with a magnetic field of up to $7 \mathrm{~T}$ applied in plane. The magnetization $M$ as a function of the magnetic field $H$ shows an "S"-shaped behavior at room temperature, as shown in Fig.1(a). The data have been corrected for the linear diamagnetic contribution of the substrate. $M$ saturates at $\mu_{0} H \simeq 3 \mathrm{~T}$ showing values of $M_{\mathrm{S}}=1.02$ and 1.95 Bohr magnetons $\left(\mu_{\mathrm{B}}\right)$ per Co atom for the samples grown at $400^{\circ} \mathrm{C}$ (green) or $500^{\circ} \mathrm{C}$ (blue), respectively. We note that the shape of the $M(H)$ curves is about the same for both deposition temperatures. Similar RT magnetization data for doped $\mathrm{ZnO}$ thin films have been reported in literature $6,8,9,10,28,30$. It is tempting to interpret these curves as evidence for RT ferromagnetism as they cannot be explained by simple paramagnetic Brillouin functions for $\mathrm{Co}^{2+}$ in the high-spin $(S=3 / 2)$ or low-spin state $(S=1 / 2)$ due to their large slopes at zero field. However, within experimental error our data do not show any remanent RT magnetization at zero field (see inset in Fig.1(a)). This observation is consistent with literature $\underline{6,30}$. This lack of any observable magnetic hysteresis makes an interpretation in terms of a dilute ferromagnetic semiconductor questionable.

As shown in Fig.1(a), it is easily possible to fit the data by a Langevin function

$$
M(B)=M_{\mathrm{S}}\left(\operatorname{coth} \frac{\mu B}{k_{\mathrm{B}} T}-\frac{k_{\mathrm{B}} T}{\mu B}\right)
$$

with the magnetic induction $B$, the Boltzmann constant $k_{\mathrm{B}}$, the measuring temperature $T=300 \mathrm{~K}$, and the moment $\mu$ of (super-)paramagnetic particles within the thin film. Fitting the data (solid lines in Fig.1(a)) gives $\mu=2370 \mu_{\mathrm{B}}$ and $5910 \mu_{\mathrm{B}}$ for the films grown at $400^{\circ} \mathrm{C}$ and $500^{\circ} \mathrm{C}$, respectively. The good fits suggest that the measured magnetization curves can be consistently explained by the presence of superparamagnetic particles in the ZnO matrix with average magnetic moments of $2370 \mu_{\mathrm{B}}$ and $5910 \mu_{\mathrm{B}}$, as already suggested earlier 27 . This calls into question the widely accepted interpretation of the RT magnetization data of cobaltdoped $\mathrm{ZnO}$ thin film samples. In the vast majority of publications, magnetization curves similar to those shown in Fig.1(a) have been regarded as proof for the existence of carrier mediated RT ferromagnetic coupling between dilute $\mathrm{Co}^{2+}$ moments in the $\mathrm{ZnO}$ matrix. However, the perfect fit of the data by a Langevin function and the very small or even absent remanent magnetization shows that an alternative interpretation of the magnetization curves 
in terms of nanometer sized superparamagnetic particles with average moments of a few $1000 \mu_{B}$ may be more adequate. We note that even a finite remanence would be consistent with this interpretation since clusters with larger diameter may be blocked already at RT. With the saturation magnetization of $1.7 \mu_{\mathrm{B}} /$ Co for metallic Co at room temperature ${ }^{34}$ and assuming a hexagonal crystallographic structure, the diameter of metallic Co clusters in our samples is determined to about $3 \mathrm{~nm}\left(T_{G}=400^{\circ} \mathrm{C}\right)$ and $4 \mathrm{~nm}\left(500^{\circ} \mathrm{C}\right)$ to yield the moments given above.

To further clarify the nature of magnetism in our cobalt-doped ZnO films we have performed zero field- (ZFC) and field-cooled (FC) measurements of the temperature dependence of the magnetization. The results are presented in Fig.1(b), where we have plotted $M(T)$ for the samples cooled down from room temperature to $4 \mathrm{~K}$ at $0 \mathrm{~T}(\mathrm{ZFC})$ or $7 \mathrm{~T}(\mathrm{FC})$, respectively. Then, $M(T)$ was measured while warming up the sample at a small measuring field of $10 \mathrm{mT}$. For both samples, there is a clear difference between the ZFC and the FC data at low temperatures. In particular, the ZFC curves show pronounced maxima at around $15 \mathrm{~K}$ and $38 \mathrm{~K}$ for the samples grown at $400^{\circ} \mathrm{C}$ or $500^{\circ} \mathrm{C}$, respectively. These maxima which are absent in the FC measurements might originate from domain formation in a ferromagnetic ZnO:Co thin film. However, more likely they can be explained in terms of the blocking of superparamagnetic metallic Co nanoparticles within a diamagnetic ZnO matrix ${ }^{35}$. This explanation is confirmed by plotting the data from zero field-cooling as a function of the inverse temperature up to $375 \mathrm{~K}$ (see inset of Fig.1(b)). Well above the blocking temperature, the magnetization straightly follows the Curie law $\left(M \propto T^{-1}\right)$ valid for pure paramagnets.

Additional information can be obtained from the temperature dependence of the real part of the AC susceptibility (Fig.2). This quantity was measured on warming up the sample after cooling down in zero magnetic field. The small AC magnetic field with amplitude $\mu_{0} H_{\mathrm{AC}}=0.5 \mathrm{mT}$ and frequency $f=0.1,1$, and $10 \mathrm{~Hz}$ was applied parallel to the film plane. The $\chi_{\mathrm{AC}}^{\prime}(T)$ curves show pronounced maxima at about the same temperatures where the FC and ZFC $M(T)$ curves start to deviate from each other (cf. Fig.1). The positions of the maxima shift to higher temperatures with increasing driving frequency. This is expected if the measured $\chi_{\mathrm{AC}}^{\prime}$ signal originates from superparamagnetic particles ${ }^{36}$. A quantitative measure of the frequency shift is given by the relative shift of the peak temperature per decade shift in frequency, $\Delta T_{\mathrm{B}} / T_{\mathrm{B}} \Delta \log _{10} f$. For the samples grown at $400^{\circ} \mathrm{C}$ and $500^{\circ} \mathrm{C}$, we obtain values of 0.06 and 0.10 , respectively. According to Dormann et al. $\stackrel{36}{\underline{37}}$, these values 
point to the presence of magnetic particles in between the non-interacting and the weakly interacting regime. Additionally, the frequency dependence of $T_{\mathrm{B}}$ for our thin films can be well described using the Néel-Arrhenius law

$$
f=f_{0} \exp \left(-\frac{E_{\mathrm{a}}}{k_{\mathrm{B}} T}\right)
$$

valid for superparamagnetic particles $\frac{36}{37}$, with an activation energy $E_{\mathrm{a}}$ and a characteristic frequency $f_{0}$. Fitting the data (see insets of Fig.2), we obtained $E_{\mathrm{a}} / k_{\mathrm{B}}=580 \mathrm{~K}$ and $910 \mathrm{~K}$ for the samples grown at $400^{\circ} \mathrm{C}$ and $500^{\circ} \mathrm{C}$, respectively. The derived blocking temperatures and activation energies agree well with those expected for metallic Co nanoparticles with a diameter of 3-4 $\mathrm{nm}^{\underline{34}, 38}$. Evidently, both DC and AC magnetization measurements point to the existence of superparamagnetic particles within our $\mathrm{Zn}_{0.95} \mathrm{Co}_{0.05} \mathrm{O}$ thin films. Unfortunately, in the vast majority of literature neither the FC and ZFC magnetization curves nor the AC susceptibility data are shown so that it is difficult to rule out superparamagnetism.

A powerful tool for clarifying the microscopic origin of ferromagnetism is x-ray magnetic circular dichroism (XMCD) spectroscopy. This element specific technique allows to obtain microscopic information on the magnetic ordering of the Co magnetic moments alone. We performed x-ray absorption near edge spectroscopy (XANES) and XMCD at the European synchrotron radiation facility (ESRF) in Grenoble (France), beamline ID08, at temperatures of $10 \mathrm{~K}$ and $300 \mathrm{~K}$. Magnetic fields of up to $4 \mathrm{~T}$ were applied parallel or anti-parallel to the incident x-ray beam. Two APPLE II undulators provide a left (lcp) or right circular polarization (rcp) of almost 100\% for the incoming photons. The samples were aligned at an angle of $75^{\circ}$ between the surface normal and the incident light. Both the fluorescence yield (FY) and the total electron yield (TEY) signals were detected simultaneously in a photon energy range from 765 to $815 \mathrm{eV}$. The energy resolution of the spherical grating monochromator is $\Delta E / E=5 \times 10^{-4}$ at $850 \mathrm{eV}$. The XMCD spectra were obtained as direct difference between consecutive XANES scans at the Co $L_{3}$ and $L_{2}$ edges recorded with opposite helicities of the x-rays. Each measurement consists of eight XANES scans taken at constant magnetic field with four spectra taken for left (lcp) and four for right circularly polarized (rcp) light, respectively. As the escape depth for the secondary electrons is much shorter $\underline{\underline{39}}$ than for the fluorescence photons $\underline{\underline{40}}$, the TEY mode probes the surface of the sample while the FY mode is more sensitive to the bulk.

The XANES scans were taken at constant magnetic field for left (lcp) and right circularly 
polarized (rcp) light, respectively. The corresponding intensities are denoted by $I_{+}$(lcp) and $I_{-}$(rcp). In the following, we will focus on thin films grown at $400^{\circ} \mathrm{C}$. The data were evaluated in the following four steps: First, the XANES intensity at energies below the Co $L_{3}$ edge $(772 \mathrm{eV})$ was set to zero by subtracting a constant background. Second, at $810 \mathrm{eV}$, i.e. above the $L_{2}$ edge, the XANES intensity was normalized to unity. This is motivated by the assumption of no Co induced absorption below the $L_{3}$ edge and a constant non-resonant absorption above the $L_{2}$ edge. In this way, the spectra were corrected for time-dependent drifts in the measurement setup. Averaging all corrected spectra results in the quantity $\left(I_{+}+I_{-}\right) / 2$ shown in Figs.3(a) and (b) for FY and TEY, respectively. Third, in order to remove the non-resonant background, step functions sketched as dashed lines in Figs.3(a) and (b) were subtracted. The positions of the steps were set to the center positions of the $L_{3}$ and $L_{2}$ edges, respectively, with a fixed height ratio of $2: 1$ according to the number of states available for non-resonant absorption ${ }^{41}$. Finally, the XMCD signal was determined by subtracting the corrected rcp from the lcp XANES spectra. In Figs.3(c) and (d) the averaged difference $\left(I_{+}-I_{-}\right) / 2$ is shown for FY and TEY, respectively. We note that the TEY XMCD signal at the $L_{2}$ edge is very weak and may become smaller than the experimental error. This is consistent with literature, where calculations for $\mathrm{Co}^{2+}$ in the high spin state show only a very weak $L_{2}$ signal ${ }^{43}$. Unfortunately, this may result in the fact that there is no longer any observable sign change in the XMCD signal at the $L_{2}$ edge (Fig.3(d)). We also note that some of the step functions used for background subtraction go above the experimental XANES data (see Figs.3(a) and (b)) what might be considered unphysical. We also used step functions staying below the experimental data in the relevant energy range. However, this results in the problem that the background curve does not meet the data curve above $810 \mathrm{eV}$. A possible remedy would be the subtraction of an additional linear background which is however also difficult to justify. Since in any way the use of different background functions only results in a variation of about $30 \%$ in the derived magnetic moments, but not in the magnetic field dependence of the moments, we used the step functions shown in Figs.3(a) and (b).

Comparing the XMCD spectra for FY and TEY shows significant differences. Figs.3(e) and (f) display the region around the Co $L_{3}$ edge on an enlarged scale at room (e) and at low temperature (f), respectively. The TEY spectra exhibit a pronounced fine structure: five peaks are visible at photon energies of 779.0, 779.6, 780.2, 781.6, and $782.0 \mathrm{eV}$. The measured 
data correspond well with calculations for $\mathrm{Co}^{2+}$ (shown in black) taken from Kobayashi et $a l . \stackrel{42}{\underline{n}}$, in particular at low temperature. Recalling the fact that the typical escape depth of the secondary electrons in TEY mode is less than $10 \mathrm{~nm}^{39}$, this correspondence indicates the existence of $\mathrm{Co}^{2+}$ ions near the surface of the $\mathrm{Zn}_{0.95} \mathrm{Co}_{0.05} \mathrm{O}$ film. In contrast, there is only a very weak fine structure in the FY spectra which completely disappears at room temperature. The FY spectra are close to XMCD data published for metallic cobalt (shown in green) by Mamiya et al. $\underline{\underline{43}}$. The correspondence between the data sets is striking. As the fluorescence photons have a larger escape depth of approximately $100 \mathrm{~nm} \stackrel{40}{ }$, this observation provides further clear evidence for the presence of metallic Co precipitates in the "bulk" of $\mathrm{Zn}_{0.95} \mathrm{Co}_{0.05} \mathrm{O}$ thin films.

Applying the magnetooptical sum rules $\underline{44} \underline{45}$, we have derived the effective spin moment $m_{\mathrm{s}, \text { eff }}$ and orbital moment $m_{\mathrm{l}}$ of Co from the XMCD spectra for different magnetic fields following Chen's approach 11 and using $n_{3 \mathrm{~d}}=7$ for the $3 \mathrm{~d}$ electron occupation number. While the decay of the excited state via secondary electrons varies by only about $20 \%$ for the $L_{3}$ and $L_{2}$ edges the fluorescence decay may differ up to $400 \%$ due to self-absorption effects $\underline{46}$. Therefore, the FY-XANES spectra were corrected prior to the application of the sum rules by scaling the $L_{3}$ signal. The scaling factor is determined at fields below $1 \mathrm{~T}$ and is given by the area ratio of $\frac{\text { XANES }_{\mathrm{L}_{3}}^{T E Y} / \mathrm{XANES}_{\mathrm{L}_{2}}^{\mathrm{TEY}}}{\mathrm{XANES}_{\mathrm{L}_{3}}^{\mathrm{FY}} / \mathrm{XANES}_{\mathrm{L}_{2}}^{\mathrm{FY}}}$. The results are shown in Fig.4(a) for the FY mode at room temperature. The "S"-shaped field dependence of the derived effective spin magnetic moment $m_{\mathrm{s} \text {,eff }}^{\mathrm{FY}}(H)$ clearly reminds of the $M(H)$ curves shown in Fig.1. In fact, the $m_{\mathrm{s}, \text { eff }}^{\mathrm{FY}}(H)$ and the $M(H)$ curves from the sample grown at $400^{\circ} \mathrm{C}$ match well except for a scaling factor of 2.5. The corresponding saturation magnetization derived from the FY-XMCD signal is only $0.4 \mu_{\mathrm{B}} /$ Co. This can be explained assuming the presence of a "dead magnetic layer" of some ten nanometers at the surface where the metallic cobalt clusters are oxidized. We suggest that this layer only marginally affects the overall SQUID signal of the $350 \mathrm{~nm}$ thick sample but may significantly reduce the magnetization derived from the FY-XMCD signal because of the FY-XMCD probing depth of only $100 \mathrm{~nm} \underline{40}$. In Fig.4(a), also the orbital magnetic moment $m_{\mathrm{l}}^{\mathrm{FY}}(H)$ is shown. It is aligned parallel to $m_{\mathrm{s}, \text { eff }}^{\mathrm{FY}}(H)$ with values up to about $0.07 \mu_{\mathrm{B}} /$ Co. It is obvious that $m_{1}^{\mathrm{FY}}(H)$ cannot account for the discrepancy to the saturation moment measured by SQUID magnetometry.

In the following, we will further discuss the effective spin magnetic moment. In Fig.4(b), we compare the $m_{\mathrm{s}, \text { eff }}(H)$ values obtained in the FY and TEY mode. While the FY signal 
nicely follows the overall magnetization, for the TEY mode only very small $m_{\mathrm{s} \text {,eff }}^{\mathrm{TEY}}$ values are obtained. They are comparable to the average paramagnetic moment expected for $\mathrm{Co}^{2+}$ ions in the high-spin state $(S=3 / 2)$. We would like to point out that in general the quantitative analysis of the TEY signal is difficult and involves considerable errors due to the small signal level. However, as shown in the inset of Fig.4(b), $m_{\mathrm{s} \text {, eff }}^{\mathrm{TEY}}(H)$ follows roughly the Brillouin function $B_{3 / 2}$ for $g=2, S=3 / 2$ and $T=300 \mathrm{~K}$, expected for isolated $\mathrm{Co}^{2+}$ ions. Together with the observed fine structure of the TEY-XMCD signal from Fig.3(e,f), this result confirms the presence of magnetically uncoupled $\mathrm{Co}^{2+}$ ions within the small escape depth of the secondary electrons, i.e. the surface layer of the samples. This further supports the notion that metallic Co clusters at the sample surface are oxidized. Similar results are reported from TEY-XAS measurements $\underline{9} 27$. However, our results obtained in the FY mode clearly point to the presence of metallic Co inclusions within the (larger) escape depth of the fluorescence photons, i.e. the "bulk" of the $\mathrm{Zn}_{0.95} \mathrm{Co}_{0.05} \mathrm{O}$ thin films.

\section{STRUCTURAL ANALYSIS}

Since the magnetic characterization of the $\mathrm{Zn}_{0.95} \mathrm{Co}_{0.05} \mathrm{O}$ thin films provided clear evidence for the presence of metallic Co clusters we performed a detailed microstructural analysis to directly confirm this fact. Fig.5 shows x-ray diffraction diagrams from $\mathrm{Zn}_{0.95} \mathrm{Co}_{0.05} \mathrm{O}$ thin films grown at $400^{\circ} \mathrm{C}$ and $500^{\circ} \mathrm{C}$. At first glance, the $\omega$-2 $\theta$ scans look impeccable, as expected for phase-pure $\mathrm{Zn}_{0.95} \mathrm{Co}_{0.05} \mathrm{O}$ thin films with high crystalline quality. However, as shown in the inset, an additional weak reflection in the $\omega-2 \theta$ scan appears around $2 \theta=44.2^{\circ}$ after strongly increasing the integration time up to $400 \mathrm{~s}$ per point. The position of this peak agrees best with what is expected for the (111) reflection of fcc or the (0002) reflection of hcp cobalt. Furthermore, the $\mathrm{ZnCo}_{2} \mathrm{O}_{4}(400)$ and the $\mathrm{Co}_{3} \mathrm{O}_{4}(400)$ peaks are close. Assuming that this reflection originates from metallic Co clusters as suggested by XMCD, we can use Scherrer's expression $\underline{47}$ to derive the average cluster size from the FWHM of the diffraction peak. Doing so, we obtain diameters of 2.2 and $3.4 \mathrm{~nm}$ for the samples grown at $400^{\circ} \mathrm{C}$ and $500^{\circ} \mathrm{C}$, respectively. These values agree well with the values 3 and $4 \mathrm{~nm}$ obtained earlier from the fits of the $M(H)$ curves by Langevin functions.

To complete the microstructural analysis, we have performed a detailed transmission electron microscopy (TEM) study of the thin film samples in cross section. TEM was carried 
out using a Philips CM300UT field-emission transmission electron microscope (FEG-TEM) equipped with an electron energy imaging filter (GIF, Gatan Inc.). The images shown here were calculated as averages of two single exposures, each taken with an exposure time of $80 \mathrm{~s}$. The bright field TEM image of $\mathrm{Zn}_{0.95} \mathrm{Co}_{0.05} \mathrm{O}$ grown at $500^{\circ} \mathrm{C}$ (Fig.6(a)) shows characteristic contrasts spread all over the deposited film. The contrasts definitely do not originate from the ion milling process used for TEM sample preparation. The comparison with pure $\mathrm{ZnO}$ thin films grown under the same conditions indicates that those defects are correlated with the incorporation of $\mathrm{Co}$ into the $\mathrm{ZnO}$ film. The regions with contrast different from that of $\mathrm{ZnO}$ are on a typical scale of $5 \mathrm{~nm}$ (yellow circles). Analysis yields the observed contrast to be a typical Moiré contrast originating from overlapping crystals with different structure which can be contributed to metallic cobalt with orientation like $\mathrm{ZnO}$.

The chemical composition of those regions was evaluated using energy-filtering TEM (EFTEM). Using the three-window method $\underline{48}$ at the Co-L ionisation edge, the Co distribution map of the same region as shown in Fig.6(a) is generated (Fig.6(b)). A significant cobalt enrichment is observed exactly in the regions of the Moiré contrasts whereas the Co signal in the $\mathrm{ZnO}$ matrix is below noise level (Fig.6(b)). Also the shape and the size of these Co enriched regions clearly correlate with the structural features seen in the bright field image. Our HRTEM results provide direct evidence for the presence of Co clusters in cobalt-doped $\mathrm{ZnO}$ thin films. In order to distinguish whether the clusters consist of metallic Co or some cobalt oxide we have performed additional EFTEM studies of zinc and oxygen. The elemental maps generated at both the Zn-L edge and the O-K edge show a depletion of the corresponding elements in the regions of the Co rich clusters. The observed decrease of the $\mathrm{O}$ and the $\mathrm{Zn}$ signals in these regions further supports our conclusion that there are clusters consisting of metallic Co embedded in the cobalt-doped $\mathrm{ZnO}$ film. We may note that the HRTEM analysis yields a typical diameter of $5 \mathrm{~nm}$ for the Co cluster size in the film grown at $500^{\circ} \mathrm{C}$. This again corroborates the values derived from both the magnetic characterization and the x-ray diffraction diagrams.

\section{CONCLUSIONS}

From our comprehensive study of both the magnetic and structural properties of epitaxial $\mathrm{Zn}_{0.95} \mathrm{Co}_{0.05} \mathrm{O}$ thin films we can draw several important conclusions regarding the nature of 
magnetism in these films. First, the macroscopic magnetization obtained by SQUID magnetometry, the temperature dependence of the magnetization in the FC and ZFC mode, as well as the AC susceptibility can be consistently explained by superparamagnetic particles with magnetic moments of several $1000 \mu_{B}$. Second, the XMCD spectra obtained in TEY mode show a multiplet structure pointing to the existence of $\mathrm{Co}^{2+}$ ions in the surface layer of the samples. However, in FY mode probing the bulk of the films the spectra are smooth, resembling those of metallic cobalt. This indicates the presence of metallic cobalt inclusions in the $\mathrm{Zn}_{0.95} \mathrm{Co}_{0.05} \mathrm{O}$ thin films which most likely are oxidized in the surface region. Third, the effective spin magnetic moment for Co derived from the FY mode spectra shows the same magnetic field dependence as the macroscopic magnetization measured by SQUID magnetometry. That is, the detailed magnetic characterization of our $\mathrm{Zn}_{0.95} \mathrm{Co}_{0.05} \mathrm{O}$ films provides convincing evidence that the room-temperature ferromagnetic-like behavior results from superparamagnetic metallic Co clusters with diameters between 3 and $4 \mathrm{~nm}$. We have no evidence for bulk room-temperature ferromagnetism resulting from carrier mediated ferromagnetic exchange between diluted Co moments. The interpretation of the nature of the ferromagnetic-like behavior in $\mathrm{Zn}_{0.95} \mathrm{Co}_{0.05} \mathrm{O}$ in terms of superparamagnetic Co clusters is confirmed by our detailed microstructural analysis. Both x-ray diffractometry and HRTEM in combination with EFTEM directly prove the existence of metallic Co nanoparticles with the same diameter as derived from the magnetic characterization.

In summary, we identify metallic precipitates in $\mathrm{Zn}_{0.95} \mathrm{Co}_{0.05} \mathrm{O}$ thin films as superparamagnetic cobalt clusters of nanometer size. We argue that the magnetic behavior in our epitaxial cobalt-doped $\mathrm{ZnO}$ films is dominated by these nanosized metallic Co clusters, leading to a ferromagnetic-like response at room-temperature. The clusters are difficult to detect and can be revealed only by a systematic element specific characterization. To this end, FYXMCD and thorough microstructural analysis are particularly valuable. We emphasize that the formation and growth of the nanoparticles can be controlled by the growth conditions and co-doping. In this way, it may be possible to engineer the nanoparticles in a bottom-up technique and use them to tailor material properties for specific applications as proposed recently for Cr-rich ferromagnetic clusters in $(\mathrm{Zn}, \mathrm{Cr}) \mathrm{Te}^{26}$. 


\section{Acknowledgements}

We thank Andreas Erb for the careful preparation of the polycrystalline target materials for the pulsed laser deposition process. This work was supported by the DFG via SPP 1157 (projects GR 1132/13 and MA 1020/11), SPP 1285 (project GR 1132/14), and by the ESRF (project HE-2089). Financial support of the German Excellence Initiative via the Nanosystems Initiative Munich (NIM) is gratefully acknowledged.

* Electronic address: Matthias.Opel@wmi.badw.de

$\dagger$ Electronic address: Rudolf.Gross@wmi.badw.de

1 S.A. Wolf, D.D. Awschalom, R.A. Buhrman, J.M. Daughton, S. von Molnár, M.L. Roukes, A.Y. Chtchelkanova, and D.M. Treger, Science 294, 1488 (2001).

2 I. Žutić, J. Fabian, and S. Das Sarma, Rev. Mod. Phys. 76, 323 (2004).

3 T. Dietl, H. Ohno, F. Matsukara, J. Cibert, and D. Ferrand, Science 287, 1019 (2000).

4 H. Munekata, H. Ohno, S. von Molnar, A. Segmüller, L.L. Chang, and L. Esaki, Phys. Rev. Lett. 63, 1849 (1989).

5 H. Ohno, A. Shen, F. Matsukura, A. Oiwa, A. Endo, S. Katsumoto, and Y. Iye, Appl. Phys. Lett. 69, 363 (1996).

6 M. Venkatesan, C.B. Fitzgerald, J.-G. Lunney, and J.M.D. Coey, Phys. Rev. Lett. 93, 177206 (2004).

7 K. Rode, A. Anane, R. Mattana, J.-P. Contour, O. Durand, and R. LeBourgeois, J. Appl. Phys. 93, $7676(2003)$.

8 D. Chakraborti, J. Narayan, and J.T. Prater, Appl. Phys. Lett. 90, 062504 (2007).

9 G.L. Liu, Q. Cao, J.X. Deng, P.F. Xing, Y.F. Tian, Y.X. Chen, S.S. Yan, and L.M. Mei, Appl. Phys. Lett. 90, 052504 (2006).

10 H. Pan, J.B. Yi, L. Shen, R.Q. Wu, J.H. Yang, J.Y. Lin, Y.P. Feng, J. Ding, L.H. Van, and J.H. Yin, Phys. Rev. Lett. 99, 127201 (2007).

11 K. Nielsen, S. Bauer, M. Lübbe, S.T.B. Goennenwein, M. Opel, J. Simon, W. Mader, and R. Gross, phys. stat. sol. (a) 203, 3581 (2006).

12 S.A. Chambers, T.C. Droubay, C.M. Wang, K.M. Rosso, S.M. Heald, D.A. Schwartz, K.R. 
Kittilstved, and D.R. Gamelin, Materials Today 9(11), 28 (2006).

13 K.R. Kittilstved, D.A. Schwartz, A.C. Tuan, S.M. Heald, S.A. Chambers, and D.R. Gamelin, Phys. Rev. Lett. 97, 037203 (2006).

14 J.M.D. Coey, M. Venkatesan, and C.B. Fitzgerald, Nature Mater. 4, 173 (2005).

15 K.R. Kittilstved, J. Zhao, W.K. Liu, J.D. Bryan, D.A. Schwartz, and D.R. Gamelin, Appl. Phys. Lett. 89, 062510 (2006).

16 W.K. Liu, G.M. Salley, and D.R. Gamelin, J. Phys. Chem. B 109, 14486 (2005).

17 K.R. Kittilstved, W.K. Liu, and D.R. Gamelin, Nature Mater. 5, 291 (2006).

18 S. Kolesnik, B. Dabrowski, and J. Mais, J. Appl. Phys. 95, 2582 (2004).

19 W. Pacuski, D. Ferrand, J. Cibert, C. Deparis, J.A. Gaj, P. Kossacki, and C. Morhain, Phys. Rev. B 73, 035214 (2006).

20 S. Yin, M.X. Xu, L. Yang, J.F. Liu, H. Rösner, H. Hahn, H. Gleiter, D. Schild, S. Doyle, T. Liu, T.D. Hu, E. Takayama-Muromachi, and J.Z. Jiang, Phys. Rev. B 73, 224408 (2006).

21 P. Sati, C. Deparis, C. Morhain, S. Schäfer, and A. Stepanov, Phys. Rev. Lett. 98, 137204 (2007).

22 G.S. Chang, E.Z. Kurmaev, D.W. Boukhvalov, L.D. Finkelstein, S. Colis, T.M. Pedersen, A. Moewes, and A. Dinia, Phys. Rev. B 75, 195215 (2007).

23 S. Gallego, J.I. Bertrán, J. Cerdá, and M.C. Muñoz, J. Phys.: Condens. Matter 17, L451 (2005).

24 M. Venkatesan, C.B. Fitzgerald, and J.M.D. Coey, Nature (London) 430, 630 (2004).

25 N.H. Hong, J. Sakai, N. Poirot, and V. Brizé, Phys. Rev. B 73, 132404 (2006).

26 S. Kuroda, N. Nichizawa, K. Takita, M. Mitome, Y. Bando, K. Osuch, and T. Dietl, Nature Mater. 6, 440 (2007).

27 S.C. Wi, J.-S. Kang, J.H. Kim, S.-B. Cho, B.J. Kim, S. Yoon, B.J. Suh, S.W. Han, K.H. Kim, K.J. Kim, B.S. Kim, H.J. Song, H.J. Shin, J.H. Shim, and B.I. Min, Appl. Phys. Lett. 84, 4233 (2004).

28 S. Zhou, K. Potzger, G. Zhang, F. Eichhorn, W. Skorupa, M. Helm, and J. Fassbender, J. Appl. Phys. 100, 114304 (2006).

29 X.Z. Li, J. Zhang, and D.J. Sellmyer, Solid State Commun. 141, 398 (2007).

30 C. Sudakar, J.S. Thakur, G. Lawes, R. Naik, and V.M. Naik, Phys. Rev. B 75, 054423 (2007).

31 S. Ahlers, D. Bougeard, N. Sircar, G. Abstreiter, A. Trampert, M. Opel, and R. Gross, Phys. Rev. B 74, 214411 (2006). 
C. Jaeger, C. Bihler, T. Vallaitis, S.T.B. Goennenwein, M. Opel, R. Gross, and M.S. Brandt, Phys. Rev. B 74, 045330 (2006).

33 R. Gross, J. Klein, B. Wiedenhorst, C. Höfener, U. Schoop, J.B. Philipp, M. Schonecke, F. Herbstritt, L. Alff, Yafeng Lu, A. Marx, S. Schymon, S. Thienhaus, and W. Mader, in Superconducting and Related Oxides: Physics and Nanoengineering IV, edited by D. Pavuna \& I. Bosovic (SPIE Conf. Proc., Vol. 4058, 2000), p. 278.

34 R.C. O'Handley, Modern Magnetic Materials (Wiley, New York, 2000), p. 99.

35 L. Néel, Rev. Mod. Phys. 25, 293 (1953).

36 J.L. Dormann, D. Fiorani, and E. Tronc, Adv. Chem. Phys. 98, 283 (1997).

37 J.L. Dormann, D. Fiorani, and E. Tronc, J. Magn. Magn. Mater. 202, 251 (1999).

38 F. Luis, J.M. Torres, L.M. García, J. Bartolomé, J. Stankiewicz, F. Petroff, F. Fettar, J.-L. Maurice, and A. Vaurès, Phys. Rev. B 65, 094409 (2002).

39 S.J. Naftel and T.K. Sham, Journal of Sychrotron Radiation 6, 526 (1999).

40 B.L. Henke, P. Lee, T.J. Tanaka, R.L. Shimabukuro, and B.K. Fujikawa, At. Data Nucl. Data Tables 27, 1 (1982).

41 C.T. Chen, Y.U. Idzerda, H.-J. Lin, N.V. Smith, G. Meigs, E. Chaban, G.H. Ho, E. Pellegrin, and F. Sette, Phys. Rev. Lett. 75, 152 (1995).

42 M. Kobayashi, Y. Ishida, J. Hwang, T. Mizokawa, A. Fujimori, K. Mamiya, J. Okamoto, Y. Takeda, T. Okane, Y. Saitoh, Y. Muramatsu, A. Tanaka, H. Saeki, H. Tabata, and T. Kawai, Phys. Rev. B 72, 201201(R) (2005).

43 K. Mamiya, T. Koide, A. Fujimori, H. Tokano, H. Manaka, A. Tanaka, H. Toyosaki, T. Fukumura, and M. Kawasaki, Appl. Phys. Lett. 89, 062506 (2006).

44 B.T. Thole, P. Carra, F. Sette, and G. van der Laan, Phys. Rev. Lett. 68, 1943 (1992).

45 P. Carra, B.T. Thole, M. Altarelli, and X. Wang, Phys. Rev. Lett. 70, 694 (1993).

46 F.M.F. De Groot, M.A. Arrio, P. Sainctavit, C. Cartier, and C.T. Chen, Solid State Commun. 92, 991 (1994).

47 B.D. Cullity and S.R. Stock, Elements of $x$-ray diffraction (3rd ed., Prentice Hall, New Jersey, 2001), p. 167.

48 F. Hofer, P. Warbichler, and W. Grogger, Ultramicroscopy 59, 15 (1995). 

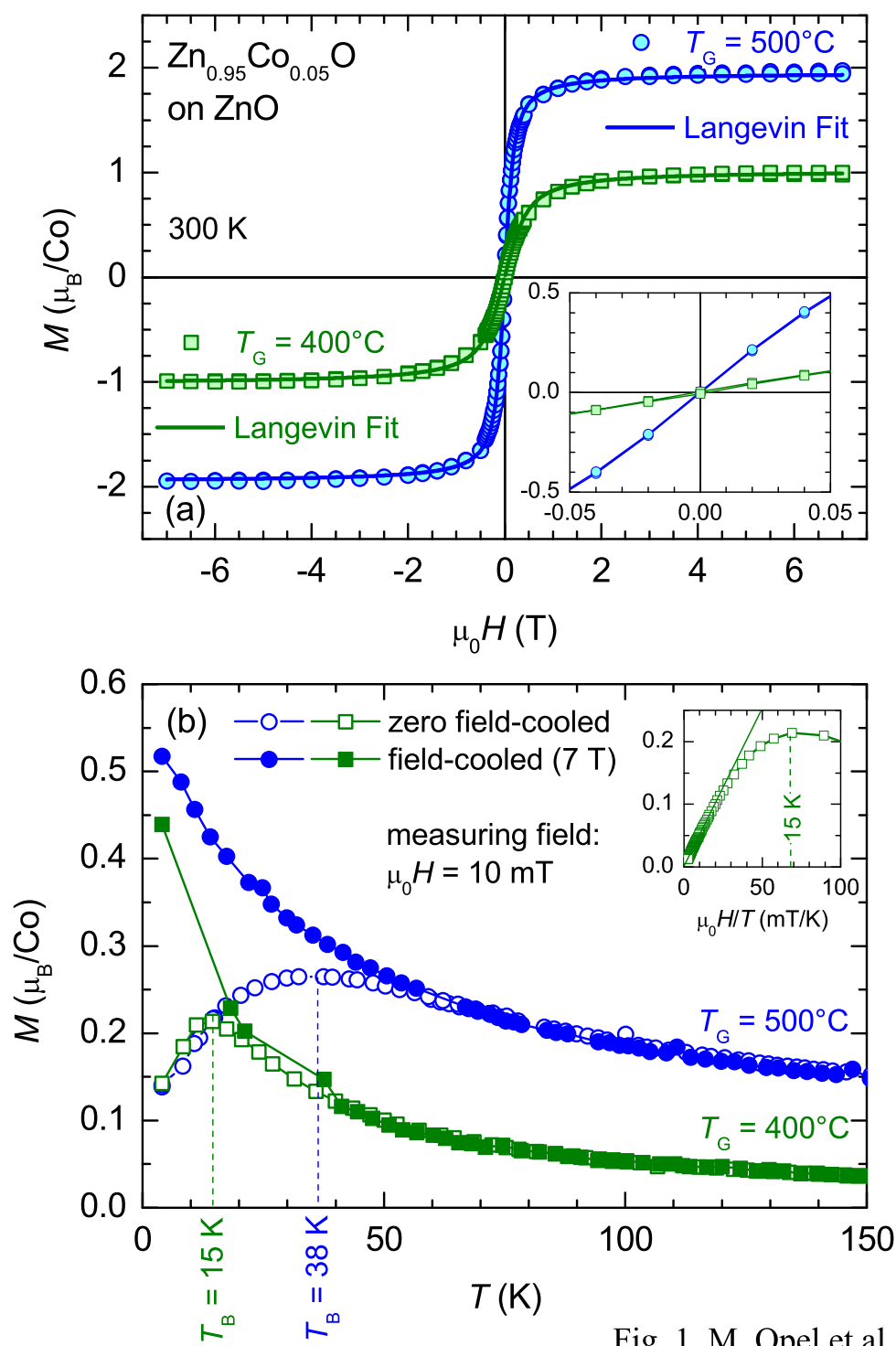

Fig. 1, M. Opel et al.

FIG. 1: (color online) (a) Room temperature magnetization curves from $\mathrm{Zn}_{0.95} \mathrm{Co}_{0.05} \mathrm{O}$ thin films grown at $T_{\mathrm{G}}=400^{\circ} \mathrm{C}$ (green squares) and $500^{\circ} \mathrm{C}$ (blue circles) with the magnetic field applied parallel to the film plane. The data can be fitted using the standard Langevin function of eq. (1) with $\mu=2370 \mu_{\mathrm{B}}$ (green line) and $5910 \mu_{\mathrm{B}}$ (blue line), respectively, indicating the presence of superparamagnetic particles in the samples. The inset shows the region around zero field on an enlarged scale. (b) Zero field-cooled (open symbols) and field-cooled magnetization measurements (closed symbols), taken at $\mu_{0} H=10 \mathrm{mT}$ as a function of temperature $T$. For both samples, the curves obtained after zero field-cooling show maxima at $T_{\mathrm{B}}=15 \mathrm{~K}$ and $38 \mathrm{~K}$, respectively, pointing to a blocking of superparamagnetic particles at these temperatures. The inset shows the zero field-cooled magnetization vs $\mu_{0} H / T$ for one sample for temperatures up to $375 \mathrm{~K}$. The data nicely follow a Curie law (straight line) for temperatures well above $T_{\mathrm{B}}$. 


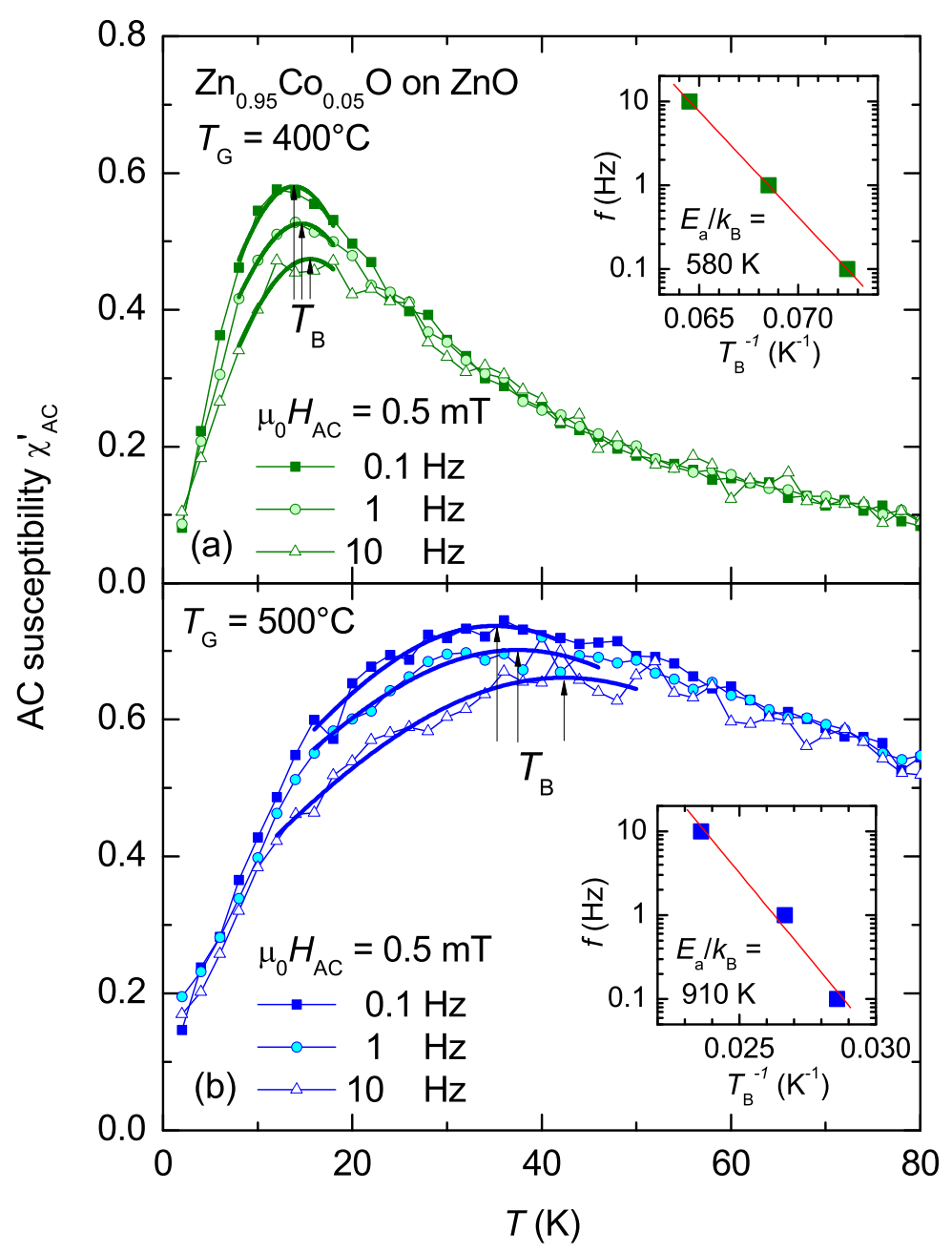

Fig. 2, M. Opel et al.

FIG. 2: (color online) Real part $\chi_{\mathrm{AC}}^{\prime}$ of the AC susceptibility (symbols) as a function of temperature $T$ from $\mathrm{Zn}_{0.95} \mathrm{Co}_{0.05} \mathrm{O}$ thin films grown at (a) $T_{\mathrm{G}}=400^{\circ} \mathrm{C}$ (green) and (b) $500^{\circ} \mathrm{C}$ (blue). The lines are guides to the eye. The $\mathrm{AC}$ field of $0.5 \mathrm{mT}$ was applied parallel to the film plane at frequencies $f=0.1,1,10 \mathrm{~Hz}$. The positions of the maxima of the $\chi_{\mathrm{AC}}^{\prime}(T)$ curves indicate the blocking temperature $T_{\mathrm{B}}$ (arrows). The insets show the frequency dependence of $T_{\mathrm{B}}$ (solid squares), which follows a Néel-Arrhenius law according to eq. (2) (straight lines). 


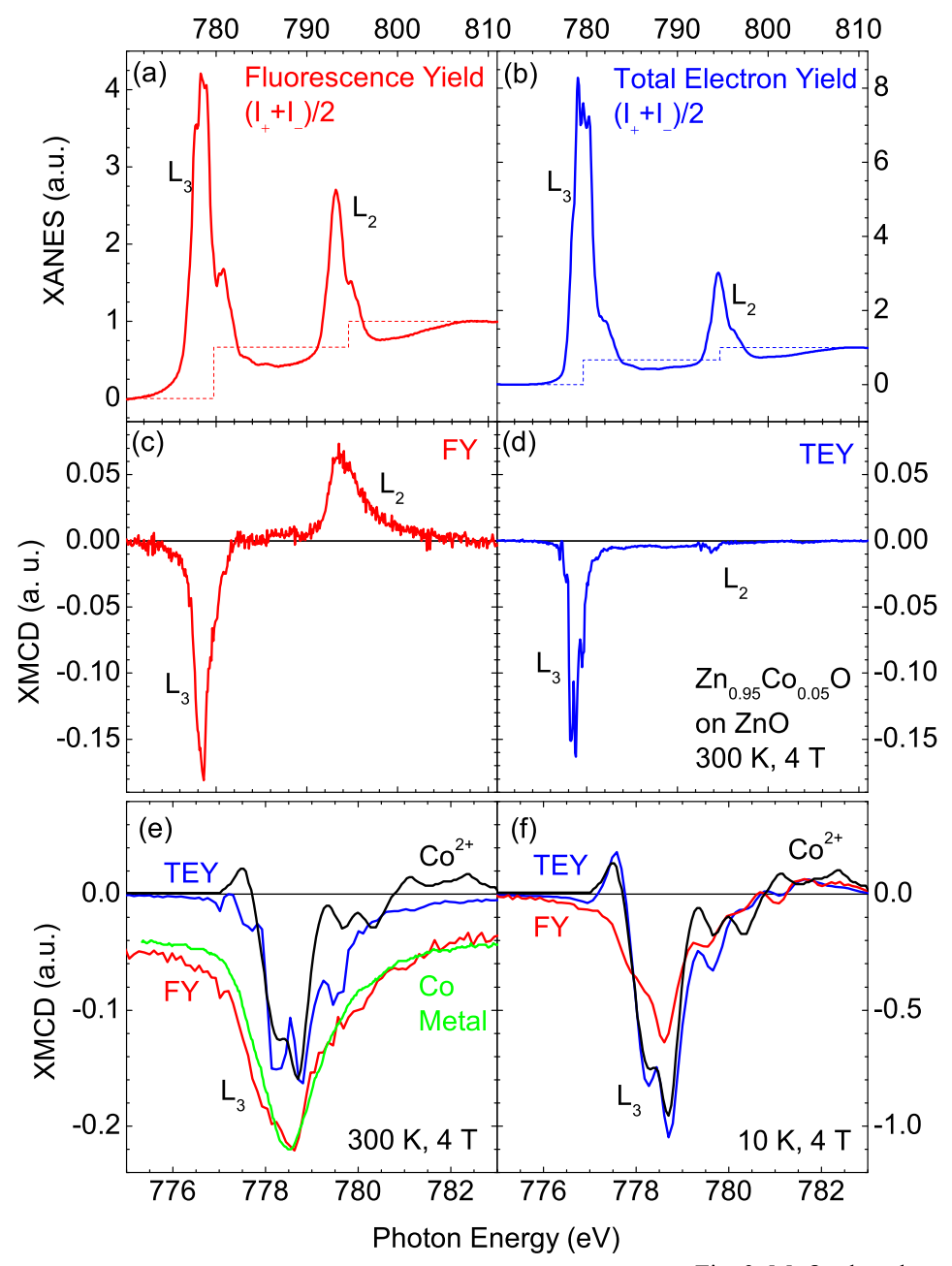

Fig. 3, M. Opel et al.

FIG. 3: (color online) XANES spectra ((a) and (b)) and XMCD spectra ((c) and (d)) of a $\mathrm{Zn}_{0.95} \mathrm{Co}_{0.05} \mathrm{O}$ film grown at $T_{\mathrm{G}}=400^{\circ} \mathrm{C}$. The data were measured in the fluorescence yield mode (FY, red, left panels) and total electron yield mode (TEY, blue, right panels), respectively, at $300 \mathrm{~K}$ and an applied magnetic field of $4 \mathrm{~T}$. (e) and (f) show the region around the $L_{3}$ edge on an enlarged scale together with the XMCD calculated for $\mathrm{Co}^{2+}$ (black, taken from $\stackrel{42}{ }$ ) and measured for metallic cobalt (green,, 43 ). In (e), for clarity the curves for FY and Co metal have been shifted by -0.04 a.u. 


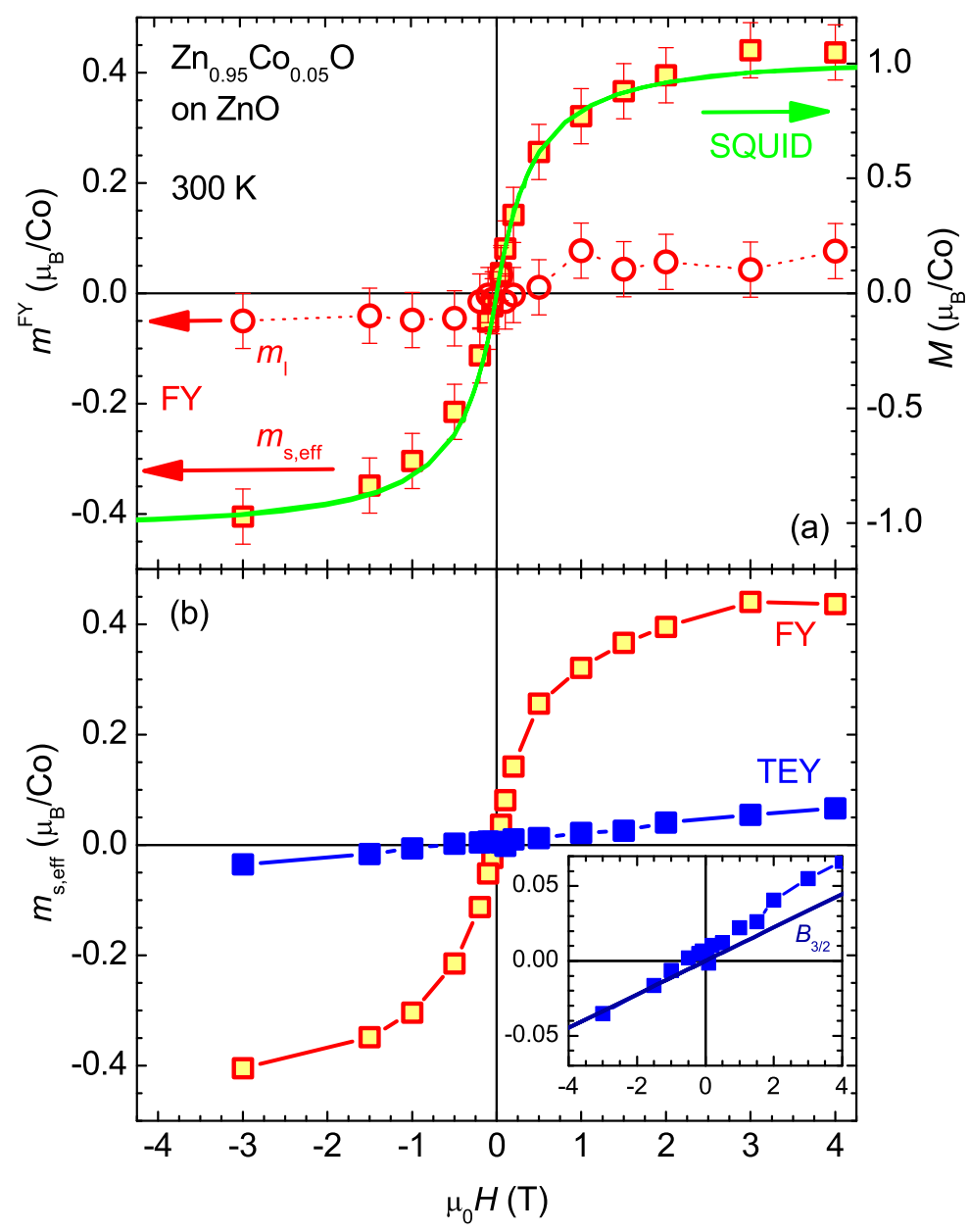

Fig. 4, M. Opel et al.

FIG. 4: (color online) (a) Room-temperature effective spin magnetic moment $m_{\mathrm{s} \text {,eff }}^{\mathrm{FY}}$ (squares) and orbital moment $m_{1}^{\mathrm{FY}}$ (circles) of Co in a $\mathrm{Zn}_{0.95} \mathrm{Co}_{0.05} \mathrm{O}$ thin film plotted versus the applied magnetic field. The moments are derived from the XMCD intensities (FY) using the magnetooptical sum rules. For comparison we also have plotted the magnetization $M(H)$ from Fig.1 measured by SQUID magnetometry (green line, right scale). (b) Effective spin magnetic moments $m_{\mathrm{s} \text {,eff }}$ derived from the XMCD spectra recorded in the FY (red) and TEY (blue) mode at $300 \mathrm{~K}$. In the inset, $m_{\mathrm{s}, \text { eff }}^{\mathrm{TEY}}(H)$ (blue) is compared to a Brillouin function (solid line) calculated for $\mathrm{Co}^{2+}$ ions $(g=2$, $S=3 / 2, T=300 \mathrm{~K})$. 


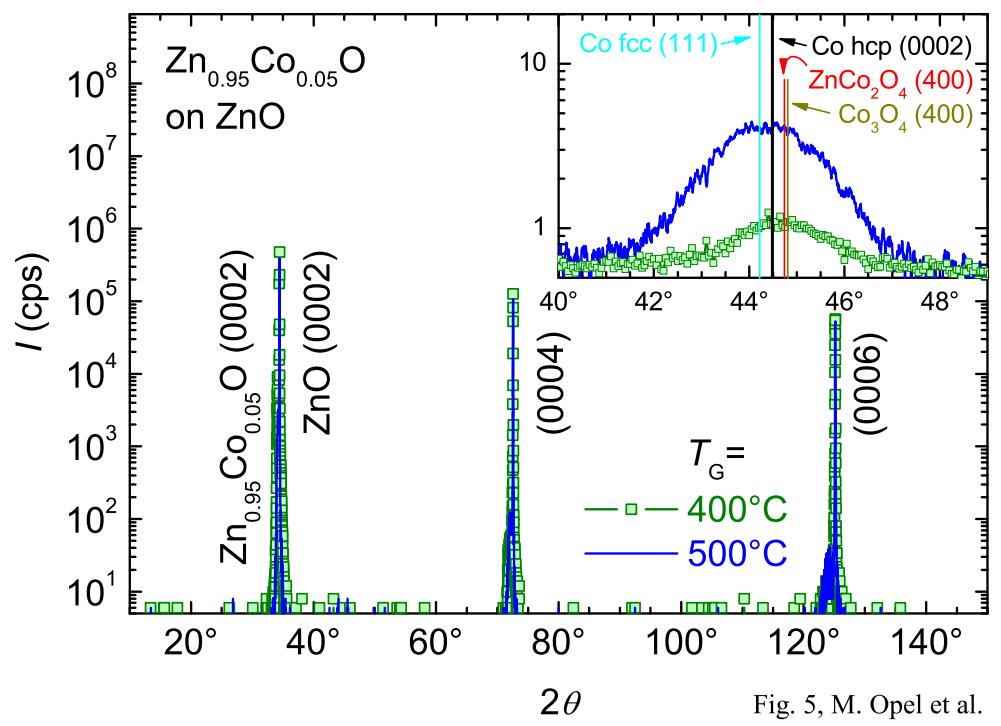

FIG. 5: (color online) X-ray diffraction diagrams ( $\omega-2 \theta$ scans) from the $\mathrm{Zn}_{0.95} \mathrm{Co}_{0.05} \mathrm{O}$ thin films grown at $400^{\circ} \mathrm{C}$ and $500^{\circ} \mathrm{C}$. The inset shows an enlargement of the region around $2 \theta=44^{\circ}$. After strongly increasing the integration time a minority phase reflection can be revealed at a position which agrees well with some peak positions expected for fcc or hcp metallic Co, $\mathrm{ZnCo}_{2} \mathrm{O}_{4}$ or $\mathrm{Co}_{3} \mathrm{O}_{4}$ (vertical lines).
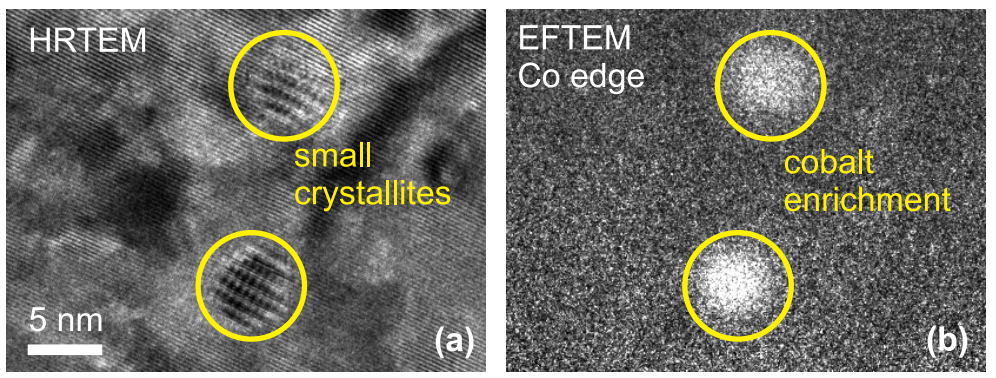

Fig. 6, M. Opel et al.

FIG. 6: (color online) (a) Bright field TEM micrograph of a $\mathrm{Zn}_{0.95} \mathrm{Co}_{0.05} \mathrm{O}$ thin film in [1]̄00] orientation grown at $500^{\circ} \mathrm{C}$. Circles highlight regions with contrast originating from clusters with crystal structure different from ZnO. (b) Elemental map of Co clearly reveals Co enrichment at locations of the clusters. 